\title{
Comparison between three different equations for the estimation of glomerular filtration rate in predicting mortality after coronary artery bypass
}

Sandro Gelsomino ${ }^{1,2^{*}+}$ (D), Massimo Bonacchi ${ }^{3 \dagger}$, Fabiana Lucà ${ }^{1,4}$, Fabio Barili ${ }^{5}$, Stefano Del Pace ${ }^{3}$, Orlando Parise ${ }^{1}$, Daniel M. Johnson ${ }^{1}$ and Michele Massimo Gulizia ${ }^{4,6}$

\begin{abstract}
Background: This study was undertaken to compare the accuracy of chronic kidney disease-epidemiology collaboration (eGFR $R_{\text {CKD-EPI }}$ ) to modification of diet in renal disease (eGFR $\mathrm{RDRD}_{\text {) }}$ ) and the Cockcroft-Gault formulas of Creatinine clearance $\left(\mathrm{C}_{\mathrm{CG}}\right)$ equations in predicting post coronary artery bypass grafting $(C A B G)$ mortality.

Methods: Data from 4408 patients who underwent isolated CABG over a 11-year period were retrieved from one institutional database. Discriminatory power was assessed using the c-index and comparison between the scores' performance was performed with DeLong, bootstrap, and Venkatraman methods. Calibration was evaluated with calibration curves and associated statistics.

Results: The discriminatory power was higher in eGFR CKD-EPI $_{\text {than }}$ eGFR $\mathrm{R}_{M D D}$ and $\mathrm{C}_{\mathrm{CG}}$ (Area under Curve [AUC]:0.77, 0.55 and 0.52, respectively). Furthermore, eGFR (AUC: 0.53) while it was not influenced by higher eGFRs, age, and body size. In contrast, the MDRD equation was accurate only in women (calibration statistics $p=0.72$ ), elderly patients $(p=0.53)$ and subjects with severe impairment of renal function ( $p=0.06$ ) whereas $C_{\mathrm{CG}}$ was not significantly biased only in patients between 40 and 59 years $(p=0.6)$ and with eGFR $45-59 \mathrm{ml} / \mathrm{min} / 1.73 \mathrm{~m}^{2}(p=0.32)$ or $\geq 60 \mathrm{ml} / \mathrm{min} / 1.73 \mathrm{~m}^{2}(p=0.48)$.

Conclusions: In general, CKD-EPI gives the best prediction of death after CABG with unsatisfactory accuracy and calibration only in patients with severe kidney disease. In contrast, the CG and MDRD equations were inaccurate in a clinically significant proportion of patients.
\end{abstract}

Keywords: Coronary artery bypass, Renal function, Glomerular filtration, Risk score

\section{Background}

Preoperative renal impairment is a well-established predictor of adverse outcomes in patients undergoing coronary artery bypass grafting (CABG) [1-3]. In addition, with advances in the fields of nephrology, cardiology, and cardiac surgery, an increasing number of patients with renal dysfunction are being offered coronary

\footnotetext{
* Correspondence: sandro.gelsomino@maastrichtuniversity.nl

${ }^{\dagger}$ Sandro Gelsomino and Massimo Bonacchi contributed equally to this work.

${ }^{1}$ Cardiothoracic Department, Maastricht University Hospital, Florence, Italy

${ }^{2}$ Department of Cardiothoracic Surgery, Cardiovascular Research Institute

Maastricht University, Universiteitssingel 50, 6229, ER, Maastricht, The

Netherlands

Full list of author information is available at the end of the article
}

revascularization [4].Therefore, accurate preoperative evaluation of renal function is recommended before CABG [5].

Estimated glomerular filtration rate (eGFR) is now considered a more sensitive marker of renal function than serum creatinine alone identifying patients with even mild renal impairment despite normal or nearly normal creatinine levels [6-8].

Different formulas to estimate eGFR have been implemented $[1,2,9]$ and, amongst these, the Modification of Diet in Renal Disease (MDRD) equation $\left(\mathrm{eGFR}_{\mathrm{MDRD}}\right)$ [1, 2, 9], the more recently defined Chronic Kidney Disease-Epidemiology Collaboration

(c) The Author(s). 2019 Open Access This article is distributed under the terms of the Creative Commons Attribution 4.0 International License (http://creativecommons.org/licenses/by/4.0/), which permits unrestricted use, distribution, and 
$\left(\mathrm{CKD}_{\mathrm{EPI}}\right)$ equation (eGFR $\left.\mathrm{CKD-EPI}\right)[10,11]$ along with the Cockcroft-Gault equation formula of Creatinine Clearance $\left(\mathrm{C}_{\mathrm{CG}}\right)[7,8,12]$ are the most widely employed.

The predictive value of eGFR on mortality and morbidity following CABG has been widely demonstrated [9, $10,11,12]$. Nonetheless, papers have concentrated on patients with serum creatinine or eGFR calculated by the $\mathrm{C}_{\mathrm{CG}}$ equation or MDRD $[2,13,14]$ and, at the best of our knowledge, no study exists comparing eGFR $\mathrm{MDRD}_{\mathrm{M}}$ and $C_{C G}$. eGFR ${ }_{\text {CKD-EPI }}$ in their predictive value of postCABG mortality.

Therefore, in this study we test the reliability of these three formulae in predicting mortality after CABG and compare their discrimination and calibration power. In addition, discrimination and calibration of the three models were also evaluated in relation to factors that may influence the absolute bias of the equations [15].

\section{Methods}

This study was performed in accordance with the Declaration of Helsinki and following STROBE guidelines [16]. Consecutive patients undergoing isolated CABG at Careggi Hospital (Florence, Italy) between 2006 and 2017 were retrospectively enrolled in the study.

\section{Definitions}

Definitions and calculations were as in our previous research [17]. Kidney dysfunction was defined following the recently updated Kidney Disease Outcomes Quality Initiative (KDOQI) [18] and Kidney Disease Improving Global Outcomes (KDIGO) Guidelines [7].

The $C_{C G}$ [19], MDRD [20] and Chronic Kidney Disease (CKD)-EPI estimate of renal function were calculated as recommended $[15,21]$ and normalized to $1.73 \mathrm{~m}^{2}$ of the body surface area (BSA) [22] and expressed in $\mathrm{ml} / \mathrm{min} / 1.73 \mathrm{~m}^{2}$. The body mass index (BMI) was calculated as body weight divided by the square of height, with body weight expressed in $\mathrm{kg}$ and height in meters.

\section{Endpoint}

The single endpoint was all-cause mortality within 30 days after CABG ( $n=3880$ cases, 79 deaths) or during index procedure hospitalization- in case of postoperative length of stay $>30$ days ( $n=528$ cases, 36 deaths) which was reported via hospital records or registry information.

\section{Statistical analysis}

Continuous data were summarized as mean and standard deviation or median and twenty-fifth to seventy-fifth percentiles in case of skewed distributions. Frequencies were reported for categorical variables. The performances of $C_{C G}$ vs. eGFR $\mathrm{CKD}_{\text {-EPI }}$ vs. eGFR $\mathrm{MDRD}_{\mathrm{M}}$ were analyzed to determine their discrimination power and calibration [23, 24]. The discrimination performance was assessed by receiver operating characteristic (ROC) and the area under the curve (AUC) with 95\% confidence intervals [25-27]. Curves were analyzed with De Long, bootstrap, and Venkatraman methods [27]. Furthermore, the model was tested by Somers' test assuming predictions as perfectly discriminating when $D_{x y}=1$ [28]. Moreover, we employed the Brier score and when it was equal to 0 the prediction could be considered perfect [29].

The calibration performance can be evaluated by generating calibration plots: the perfect calibrated predictions stay on the diagonal, whilst a curve below or above it, respectively, reflects overestimation and underestimation $[23,27,30]$.

Agreement between observed frequency and predicted probabilities were tested with the Hosmer-Lemeshow $(\mathrm{H}-\mathrm{L})$ goodness-of-fit test, whereas the comparison of actual slope and intercept with the ideal value of 1 and 0 was performed with the $U$ statistic and tested against a $x^{2}$ distribution with 2 degrees of freedom.

Discrimination and calibration performances were stratified by renal function, gender, age, body weight, and BMI due to the fact that these variables might influence the performance of the equations. Stratification of calculated eGFR $\left(\geq 60 \mathrm{ml} / \mathrm{min} / 1.73 \mathrm{~m}^{2} ; 45-59 \mathrm{ml} / \mathrm{min} /\right.$ $1.73 \mathrm{~m}^{2}, 30-44 \mathrm{ml} / \mathrm{min} / 1.73 \mathrm{~m}^{2}$ and $\leq 29 \mathrm{ml} / \mathrm{min} / 1.73 \mathrm{~m}^{2}$ ) was based on updated KDOQI and KDIGO [7, 19] and according to level of calculated EGFR, as well as on the basis of the estimates of the Cockcroft- Gault, MDRD, and CKD-EPI formulas. Using Cohen's $\mathrm{k}$ we tested the agreement between calculated and estimated EGFR .

Clinical cutoffs were used for age (18 to 39,40 to 59 , and $\geq 60$ years) [31] and body weight ( $\leq 59,60$ to 79,80 to 99 , and $\geq 100 \mathrm{~kg}$ ) [12]. Stratification for BMI followed the World Health Organization guidelines [32]. To address missing values (Additional file 1), we used fully conditional specification [33] (FCS) multiple imputation (MI) method (1000 replications).

$\mathrm{R}$, version 3.3.1 (R Foundation for Statistical Computing, Wien, Austria) with pROC, rms and Resource Selection packages was employed to carry out statistical analysis.

Significance for hypothesis testing was set at the 0.05 two-tailed level.

\section{Results}

\section{Study population}

After exclusion of subjects without an available plasma creatinine level $(n=86)$, body weight $(n=73)$ or height $(n=37)$ measurements, those undergoing preoperative dialysis $(n=18)$, who had undergone previous cardiac surgery $(n=108)$, who experienced significant (life- 
threatening) post-operative complications $(n=396)$ or with mitral insufficiency $\geq$ moderate $(n=174)$ the final population consisted of 4408 subjects who remained eligible for inclusion. Patient characteristics are presented in Table 1.

\section{Mortality}

Overall early mortality was $2.6 \%(n=115)$ : it was $24 /$ 2440 (1\%) in patients with EGFR $\geq 60 \mathrm{ml} / \mathrm{min} / 1.73 \mathrm{~m}^{2}$, 20/1170 (1.7\%) in those with EGFR between 45 and 59 $\mathrm{ml} / \mathrm{min} / 1.73 \mathrm{~m}^{2}, 39 / 703(5.5 \%)$ in subjects with EGFR ranging from 30 to $44 \mathrm{ml} / \mathrm{min} / 1.73 \mathrm{~m}^{2}$ and $32 / 95$ (33.6\%) in those with EGFR $\leq 29 \mathrm{ml} / \mathrm{min} / 1.73 \mathrm{~m}^{2}$.

\section{Overall performance}

Results of Predictive Performance, Discrimination Power and Calibration are shown in Tables 2 and 3. The c Statistic and the other measures of performance showed that only the CKD-EPI formula had any notable discriminatory power. The MDRD formula shows borderline significant discrimination, given the lower confidence limit for the C statistic is 0.50 The CG formula shows no evidence of being able to discriminate between those who died and those who did not. The ROC curves are plotted in Fig. 1 a-c: The AUC was higher in eGFR CKD-EPI than in the other two and all the comparisons amongst them showed significant differences between the three formulas with best performance by GGFR $_{\text {CKD-EPI. }}$

The pattern of calibration (Fig. $1 \mathrm{~d}-\mathrm{f}$ ) was different between the three indices. Indeed, eGFR $\mathrm{CKD}_{\mathrm{EPI}}$ was closer to the ideal line with a slight under-prediction when risk was higher but with non-significant $p$ values for the calibration statistics (both, $p=0.40$ ). In contrast, $\mathrm{eGFR}_{\mathrm{MDRD}}$ and $\mathrm{C}_{\mathrm{CG}}$ diverged significantly from the ideal diagonal with significant $\mathrm{p}$ values for the related summary statistics (both, $p=0.02$ ).

\section{Performance by kidney function}

Patients with a higher eGFR were younger $(-0.13$ years [95\%CI 0.11-1.1] per $\mathrm{ml} / \mathrm{min} / 1.73 \mathrm{~m}^{2}$ increment in eGFR) with a higher body weight $(1.64 \mathrm{~kg}$ [95\%CI $1.41-$ $2.89])$ and a higher BMI $\left(0.62 \mathrm{~kg} / \mathrm{m}^{2}\right.$ [95\%CI 0.48-0.96]). The analysis of discrimination in the subgroups demonstrated an overall worse performance of eGFR $\mathrm{MDRD}_{\text {, }}$ (Fig. 2a-c) with significant differences when eGFR was > $29 \mathrm{ml} / \mathrm{min} / 1.73 \mathrm{~m}^{2}$. In contrast, when the eGFR was $\leq 29$ $\mathrm{ml} / \mathrm{min} / 1.73 \mathrm{~m}^{2}$ the performance of MDRD was superior $(p=0.14)$. $\mathrm{C}_{\mathrm{CG}}$ showed comparable performance of

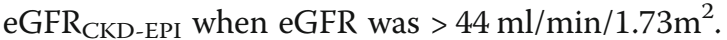

The pattern of calibration was different in the different subgroups of patients (Fig. 2 e-f: eGFR ${ }_{\text {CKD-EPI }}$ demonstrated a satisfactory calibration with eGFR $>29 \mathrm{ml} / \mathrm{min} /$ $1.73 \mathrm{~m}^{2}$ but with non-significant $p$ values for the calibration statistics $(p=0.58, p=0.78, p=0.39$, in the three
Table 1 Patient characteristics $(n=4408)$

\begin{tabular}{|c|c|}
\hline \multicolumn{2}{|l|}{ Baseline Characteristics } \\
\hline Age & $70.7[64.5-76.2]$ \\
\hline Female sex & $902(20.5)$ \\
\hline BSA & $1.85 \pm 0.17$ \\
\hline BMI & $26.2 \pm 3.8$ \\
\hline Diabetes & $793(17.9)$ \\
\hline COPD & $154(3.5)$ \\
\hline PVD & $525(11.9)$ \\
\hline CVD & $26(0.6)$ \\
\hline $\mathrm{Ml}<30$ days & $873(19.8)$ \\
\hline Unstable Angina & $1494(33.9)$ \\
\hline$\geq 3$ vessels disease & $2579(58.5)$ \\
\hline LVEF & $48.5[42.1-57.6]$ \\
\hline Creatinine (mg/dL) & $1.09[0.91-1.31]$ \\
\hline EuroScore & $8.9[4.5-12.2]$ \\
\hline STS Score & $9.9[4.8-13.1]$ \\
\hline STS PROM & $9.2[4.2-12.8]$ \\
\hline \multicolumn{2}{|l|}{ eGFR $R_{M D R D}$} \\
\hline$\geq 60$ & $2494(56.6)$ \\
\hline $45-59$ & $1200(27.2)$ \\
\hline $30-44$ & $638(14.5)$ \\
\hline$\leq 29$ & $76(1.7)$ \\
\hline \multicolumn{2}{|l|}{ eGFR } \\
\hline$\geq 60$ & $2597(58.9)$ \\
\hline $45-59$ & $1097(24.9)$ \\
\hline $30-44$ & $633(14.4)$ \\
\hline$\leq 29$ & $81(1.8)$ \\
\hline \multicolumn{2}{|l|}{$\mathrm{C}_{\mathrm{CG}}$} \\
\hline$\geq 60$ & $2440(55.4)$ \\
\hline $45-59$ & $1170(26.5)$ \\
\hline $30-44$ & $703(15.9)$ \\
\hline$\leq 29$ & $95(2.2)$ \\
\hline \multicolumn{2}{|c|}{ Operative Characteristics } \\
\hline $1 \mathrm{graft}$ & $264(6.0)$ \\
\hline 2 grafts & $1943(44.1)$ \\
\hline$\geq 3$ grafts & $2201(49.9)$ \\
\hline$O P C A B$ & $1983(44.9)$ \\
\hline
\end{tabular}

Data are shown as mean \pm SD or numbers (percentage) or median [Interquartile range]. Abbreviations. BSA: Body Surface Area; BMI: Body Mass Index; COPD: Chronic Obstructive Pulmonary Disease; PVD: Peripheral Vascular Disease; CVD: Cerebrovascular Disease; MI: Myocardial Infarction; LVEF: Left Ventricular Ejection Fraction; STS: Society of Thoracic Surgeons; PROM: Predicted risk of mortality; eGFRMDRD: Glomerular Filtration Rate estimated by the Modification in Diet in Renal Disease; eGFRCKD-EPI: $(\mathrm{ml} / \mathrm{min} / 1.73 \mathrm{~m} 2)$ Glomerular Filtration Rate estimated by Chronic Kidney Disease-Epidemiology Collaboration equation $(\mathrm{ml} / \mathrm{min} / 1.73 \mathrm{~m} 2)$; CCG: Creatinine Clearance estimated by Cockroft-Gault formula (ml/min/1.73 m2); OPCAB: Off-Pump Coronary Artery Bypass 
Table 2 Predictive performance and discrimination power

\begin{tabular}{|c|c|c|c|c|c|c|c|c|c|}
\hline & & eGFR & AUC & $95 \% \mathrm{Cl}$ & De Long & Bootstrap & Venkatraman & Somers & Brier \\
\hline \multirow[t]{3}{*}{$\overline{\text { All }}$} & & CKD-EPI & 0.77 & $0.73-0.81$ & $<0.01^{*}$ & $<0.01^{*}$ & $<0.01^{*}$ & 0.54 & $\overline{0.01}$ \\
\hline & & MDRD & 0.55 & $0.50-0.61$ & $0.65^{\dagger}$ & $0.61^{+}$ & $0.76^{\dagger}$ & 0.11 & 0.02 \\
\hline & & CG & 0.52 & $0.47-0.57$ & $<0.01^{\ddagger}$ & $<0.01^{\ddagger}$ & $<0.01^{\ddagger}$ & 0.04 & 0.02 \\
\hline \multirow[t]{12}{*}{ eGFR } & $>=60 \mathrm{ml} / \mathrm{min} . / 1.73 \mathrm{~m}^{2}$ & CKD-EPI & 0.78 & $0.73-0.82$ & $<0.01^{*}$ & $<0.01^{*}$ & $<0.01^{*}$ & 0.55 & 0.01 \\
\hline & & MDRD & 0.57 & $0.50-0.63$ & $<0.01^{\dagger}$ & $<0.01^{\dagger}$ & $<0.01^{\dagger}$ & 0.13 & 0.02 \\
\hline & & $C G$ & 0.73 & $0.69-0.75$ & $0.57^{\ddagger}$ & $0.54^{\ddagger}$ & $0.60^{\ddagger}$ & 0.44 & 0.01 \\
\hline & $45-59 \mathrm{ml} / \mathrm{min} . / 1.73 \mathrm{~m}^{2}$ & CKD-EPI & 0.67 & $0.57-0.77$ & $<0.01^{*}$ & $<0.01^{*}$ & $<0.01^{*}$ & 0.34 & 0.01 \\
\hline & & MDRD & 0.59 & $0.49-0.69$ & $<0.01^{\dagger}$ & $<0.01^{\dagger}$ & $<0.01^{\dagger}$ & 0.18 & 0.02 \\
\hline & & CG & 0.65 & $0.60-0.69$ & $0.67^{\ddagger}$ & $0.66^{\ddagger}$ & $0.69^{\ddagger}$ & 0.30 & 0.01 \\
\hline & $30-44 \mathrm{ml} / \mathrm{min} . / 1.73 \mathrm{~m}^{2}$ & CKD-EPI & 0.63 & $0.55-0.71$ & $0.04^{*}$ & $0.04^{*}$ & $0.04^{*}$ & 0.25 & 0.03 \\
\hline & & MDRD & 0.53 & $0.40-0.67$ & $0.45^{+}$ & $0.43^{+}$ & $0.55^{+}$ & 0.07 & 0.05 \\
\hline & & $C G$ & 0.57 & $0.43-0.71$ & $0.04^{\ddagger}$ & $0.04^{\ddagger}$ & $0.04^{\ddagger}$ & 0.15 & 0.04 \\
\hline & $<=29 \mathrm{ml} / \mathrm{min} .11 .73 \mathrm{~m}^{2}$ & CKD-EPI & 0.53 & $0.35-0.71$ & $0.35^{*}$ & $0.32^{*}$ & $0.54^{*}$ & 0.06 & 0.13 \\
\hline & & MDRD & 0.65 & $0.48-0.82$ & $0.10^{\dagger}$ & $0.09^{\dagger}$ & $0.16^{\dagger}$ & 0.30 & 0.14 \\
\hline & & $C G$ & 0.49 & $0.27-0.71$ & $0.23^{\ddagger}$ & $0.20^{\ddagger}$ & $0.28^{\ddagger}$ & 0.02 & 0.13 \\
\hline \multirow[t]{9}{*}{ Age } & AGE $>=60 y$ & CKD-EPI & 0.68 & $0.63-0.74$ & $<0.01^{*}$ & $<0.01^{*}$ & $<0.01^{*}$ & 0.37 & 0.02 \\
\hline & & MDRD & 0.57 & $0.51-0.63$ & $0.58^{\dagger}$ & $0.52^{\dagger}$ & $0.63^{\dagger}$ & 0.14 & 0.03 \\
\hline & & $C G$ & 0.54 & $0.48-0.60$ & $<0.01^{\ddagger}$ & $<0.01^{\ddagger}$ & $<0.01^{\ddagger}$ & 0.09 & 0.03 \\
\hline & AGE 40-59 y & CKD-EPI & 0.81 & $0.70-0.92$ & $<0.01^{*}$ & $<0.01^{*}$ & $<0.01^{*}$ & 0.62 & 0.02 \\
\hline & & MDRD & 0.59 & $0.48-0.70$ & $<0.01^{\dagger}$ & $<0.01^{\dagger}$ & $<0.01^{\dagger}$ & 0.17 & 0.03 \\
\hline & & $C G$ & 0.86 & $0.80-0.91$ & $0.45^{\ddagger}$ & $0.41^{\ddagger}$ & $0.52^{\ddagger}$ & 0.72 & 0.01 \\
\hline & AGE $18-39$ y & CKD-EPI & 0.82 & $0.72-0.96$ & $<0.01^{*}$ & $<0.01^{*}$ & $<0.01^{*}$ & 0.64 & 0.06 \\
\hline & & MDRD & 0.57 & $0.46-0.72$ & $0.03^{+}$ & $0.02^{+}$ & $0.04^{+}$ & 0.14 & 0.09 \\
\hline & & $C G$ & 0.47 & $0.08-0.85$ & $<0.01^{\ddagger}$ & $<0.01^{\ddagger}$ & $<0.01^{\ddagger}$ & 0.06 & 0.12 \\
\hline \multirow[t]{6}{*}{ Gender } & Male & CKD-EPI & 0.76 & $0.71-0.82$ & $<0.01^{*}$ & $<0.01^{*}$ & $<0.01^{*}$ & 0.53 & 0.01 \\
\hline & & MDRD & 0.55 & $0.49-0.61$ & $0.04^{+}$ & $0.04^{\dagger}$ & $0.04^{\dagger}$ & 0.10 & 0.02 \\
\hline & & $C G$ & 0.51 & $0.45-0.57$ & $<0.01^{\ddagger}$ & $<0.01^{\ddagger}$ & $<0.01^{\ddagger}$ & 0.03 & 0.02 \\
\hline & Female & CKD-EPI & 0.72 & $0.66-0.78$ & $<0.01^{*}$ & $<0.01^{*}$ & $<0.01^{*}$ & 0.44 & 0.02 \\
\hline & & MDRD & 0.55 & $0.43-0.66$ & $0.03^{+}$ & $0.03^{\dagger}$ & $0.04^{\dagger}$ & 0.09 & 0.04 \\
\hline & & $C G$ & 0.50 & $0.43-0.57$ & $<0.01^{\ddagger}$ & $<0.01^{\ddagger}$ & $<0.01^{\ddagger}$ & 0.01 & 0.06 \\
\hline \multirow[t]{12}{*}{ Weight } & $>=100 \mathrm{Kg}$ & CKD-EPI & 0.81 & $0.66-0.97$ & $0.01^{*}$ & $0.01^{*}$ & $0.02^{*}$ & 0.63 & 0.05 \\
\hline & & MDRD & 0.50 & $0.31-0.70$ & $0.04^{+}$ & $0.04^{+}$ & $0.04^{+}$ & 0.01 & 0.07 \\
\hline & & $C G$ & 0.48 & $0.26-0.69$ & $<0.01^{\ddagger}$ & $<0.01^{\ddagger}$ & $<0.01^{\ddagger}$ & 0.04 & 0.08 \\
\hline & $80-99$ Kg & CKD-EPI & 0.77 & $0.69-0.84$ & $<0.01^{*}$ & $<0.01^{*}$ & $<0.01^{*}$ & 0.53 & 0.01 \\
\hline & & MDRD & 0.52 & $0.41-0.62$ & $0.04^{+}$ & $0.04^{+}$ & $0.04^{\dagger}$ & 0.04 & 0.02 \\
\hline & & $C G$ & 0.50 & $0.40-0.62$ & $<0.01^{\ddagger}$ & $<0.01^{\ddagger}$ & $<0.01^{\ddagger}$ & 0.03 & 0.03 \\
\hline & $60-79 \mathrm{Kg}$ & CKD-EPI & 0.79 & $0.74-0.84$ & $<0.01^{*}$ & $<0.01^{*}$ & $<0.01^{*}$ & 0.59 & 0.01 \\
\hline & & MDRD & 0.56 & $0.49-0.63$ & $0.02^{\dagger}$ & $0.02^{+}$ & $0.03^{\dagger}$ & 0.12 & 0.02 \\
\hline & & $C G$ & 0.50 & $0.43-0.57$ & $<0.01^{\ddagger}$ & $<0.01^{\ddagger}$ & $<0.01^{\ddagger}$ & 0.01 & 0.03 \\
\hline & $<=59 \mathrm{Kg}$ & CKD-EPI & 0.69 & $0.56-0.83$ & $0.01^{*}$ & $0.03^{*}$ & $0.02^{*}$ & 0.39 & 0.03 \\
\hline & & MDRD & 0.55 & $0.41-0.69$ & $0.48^{\dagger}$ & $0.45^{\dagger}$ & $0.56^{\dagger}$ & 0.11 & 0.03 \\
\hline & & $C G$ & 0.53 & $0.38-0.68$ & $<0.01^{\ddagger}$ & $<0.01^{\ddagger}$ & $<0.01^{\ddagger}$ & 0.07 & 0.04 \\
\hline \multirow[t]{2}{*}{ BMI } & $>=30 \mathrm{Kg} / \mathrm{m}^{2}$ & CKD-EPI & 0.81 & $0.71-0.92$ & $<0.01^{*}$ & $<0.01^{*}$ & $<0.01^{*}$ & 0.63 & 0.01 \\
\hline & & MDRD & 0.53 & $0.37-0.69$ & $0.03^{+}$ & $0.03^{+}$ & $0.04^{+}$ & 0.06 & 0.02 \\
\hline
\end{tabular}


Table 2 Predictive performance and discrimination power (Continued)

\begin{tabular}{|c|c|c|c|c|c|c|c|c|}
\hline & eGFR & AUC & $95 \% \mathrm{Cl}$ & De Long & Bootstrap & Venkatraman & Somers & Brier \\
\hline & CG & 0.46 & $0.28-0.64$ & $<0.01^{\ddagger}$ & $<0.01^{\ddagger}$ & $<0.01^{\ddagger}$ & 0.06 & 0.03 \\
\hline \multirow[t]{3}{*}{$25-29 \mathrm{~kg} / \mathrm{m}^{2}$} & CKD-EPI & 0.76 & $0.70-0.82$ & $<0.01^{*}$ & $<0.01^{*}$ & $<0.01^{*}$ & 0.52 & 0.01 \\
\hline & MDRD & 0.55 & $0.47-0.63$ & $0.01^{\dagger}$ & $<0.01^{\dagger}$ & $0.02^{+}$ & 0.10 & 0.0 \\
\hline & CG & 0.45 & $0.37-0.53$ & $<0.01^{\ddagger}$ & $<0.01^{\ddagger}$ & $<0.01^{\ddagger}$ & 0.01 & 0.04 \\
\hline \multirow[t]{3}{*}{$18.5-24 \mathrm{Kg} / \mathrm{m}^{2}$} & CKD-EPI & 0.77 & $0.70-0.83$ & $<0.01^{*}$ & $<0.01^{*}$ & $<0.01^{*}$ & 0.53 & 0.01 \\
\hline & MDRD & 0.57 & $0.49-0.66$ & $0.00^{+}$ & $<0.01^{\dagger}$ & $0.01^{\dagger}$ & 0.15 & $0 . c$ \\
\hline & CG & 0.47 & $0.39-0.56$ & $<0.01^{\ddagger}$ & $<0.01^{\ddagger}$ & $<0.01^{\ddagger}$ & 0.04 & 0.04 \\
\hline \multirow[t]{3}{*}{$<=18.5 \mathrm{Kg} / \mathrm{m}^{2}$} & CKD-EPI & 0.77 & $0.62-0.92$ & $0.04^{*}$ & $0.04^{*}$ & $0.04^{*}$ & 0.54 & 0.1 \\
\hline & MDRD & 0.52 & $0.27-0.66$ & $0.65^{\dagger}$ & $0.62^{\dagger}$ & $0.70^{+}$ & 0.03 & 0.13 \\
\hline & CG & 0.53 & $0.13-0.94$ & $<0.01^{\ddagger}$ & $<0.01^{\ddagger}$ & $<0.01^{\ddagger}$ & 0.07 & 0.1 \\
\hline
\end{tabular}

Best performance for: Brier score $=0, A U C=1$, Somers' Dxy = 1. Abbreviations: eGFR: estimated Glomerular Filtration Rate; CI: Confidence Interval; CKD-EPI: Chronic Kidney Disease-Epidemiology Collaboration Formula; MDRD: Modification of Diet in Renal Disease Formula; CG: Cockroft-Gault Formula; BMI: Body Mass Index.* CKD-EPI vs MDRD; † MDRD vs CG; $\neq$ CG vs CKD-EPI

groups with eGFR $>29 \mathrm{ml} / \mathrm{min} / 1.73 \mathrm{~m}^{2}$, respectively). However, it tended to under-influence of predict mortality when eGFR was $\leq 29 \mathrm{ml} / \mathrm{min} / 1.73 \mathrm{~m}^{2}(p=0.04)$.

In contrast, $\mathrm{GGFR}_{\mathrm{MDRD}}$ was well calibrated at values of eGFR $\leq 29 \mathrm{ml} / \mathrm{min} / 1.73 \mathrm{~m}^{2}(p=0.06)$ whereas it diverged significantly from perfect calibration when eGFR was higher than $29 \mathrm{ml} / \mathrm{min} / 1.73 \mathrm{~m}^{2}(p=0.02, p=0.03, p=$ 0.04 , in the three groups with eGFR $>29 \mathrm{ml} / \mathrm{min} / 1.73 \mathrm{~m}^{2}$, respectively). Finally, $\mathrm{C}_{\mathrm{CG}}$ tended to over-prediction when eGFR was $<44 \mathrm{ml} / \mathrm{min} / 1.73 \mathrm{~m}^{2}(p=0.03)$.

\section{Performance by age}

Older patients had lower eGFR $\left(-0.93 \mathrm{ml} / \mathrm{min} / 1.73 \mathrm{~m}^{2}\right.$ [95\%CI 0.71-1.13] per yearly increment in age), had a lower body weight $(-1.73 \mathrm{~kg}$ [95\%CI $1.51-2.29])$ and a lower BMI $\left(-0.56 \mathrm{~kg} / \mathrm{m}^{2}\right.$ [95\%CI $\left.\left.0.48-0.75\right]\right)$. The AUC of the ROC curves (Fig. 3 a-c) was significantly higher for eGFR ${ }_{\text {CKD-EPI }}$ in all subgroups. $C_{C G}$ performed better the MDRD equation in the range of 40-59 years whereas it showed the worst performance of the three groups $<40$ years.

The pattern of calibration was different amongst age subgroups (Fig. $3 \mathrm{~d}$-f): eGFR CKD-EPI $_{\text {was }}$ close to the ideal diagonal in the oldest patients whereas it tended to slightly overestimate in the other age groups with nonsignificant $p$ values for the calibration statistics $(p=$ $0.69)$. The eGFR $_{M D R D}$ resulted to be well calibrated in the $\geq 60$ year- subgroup $(p=0.53)$ whereas it demonstrated a significant tendency to over-estimation in the other age subgroups (all, $p<0.05$ ). Finally, $\mathrm{C}_{\mathrm{CG}}$ tended to over-prediction in the $\geq 60$ year- and $18-39$ year-subgroups (both, $p=0.03$ ).

\section{Performance by gender}

Compared with men, woman had a lower body weight $(-11.4 \mathrm{~kg}[95 \% \mathrm{CI} 4.4-12.4])$ and a lower BMI $\left(-1.65 \mathrm{~kg} / \mathrm{m}^{2}\right.$ [95\%CI 1.14-6.65]).

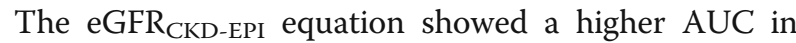
both genders (Fig. 4 a-c) with significant differences compared to the $\mathrm{C}_{\mathrm{CG}}$ and MDRD equations $(p<0.05$ for all comparisons). $\mathrm{C}_{\mathrm{CG}}$ showed a worse performance compared to eGFR $\mathrm{CDRD}_{\mathrm{MD}}$ in both genders.

In men (Fig. $4 \mathrm{~d}$-f) eGFR CKD-EPI $_{\text {reached maximum }}$ accuracy whereas it showed a tendency to overestimation in women although calibration statistics were not significant in (both, $p=0.1$ ). In contrast, $\mathrm{eGFR}_{\mathrm{MDRD}}$ was accurate in women $(p=0.72)$ and tended to overestimation in men $(p=0.03)$ whereas $C_{\mathrm{CG}}$ significantly overestimated in both sexes (both, $\mathrm{p}=0.03$ ).

\section{Performance by BMI}

Subjects with a higher BMI were younger $(-0.22$ years [95\%CI $0.09-0.44]$ each $\mathrm{kg} / \mathrm{m}^{2}$ ), had a higher body weight $(2.89 \mathrm{~kg}$ [95\%CI $2.73-3.21]$ each $\left.\mathrm{kg} / \mathrm{m}^{2}\right)$ and a higher eGFR $\left(0.78 \mathrm{ml} / \mathrm{min} / 1.73 \mathrm{~m}^{2}\right.$ [95\%CI $\left.0.56-0.91\right]$ each $\mathrm{kg} / \mathrm{m}^{2}$ ).

The AUC of ROC curves (Fig. 5 a-c) were higher

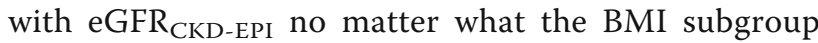
was ( $p<0.05$ for all comparisons). The calibration curves are shown in Fig. 5 D-F: the eGFR CKD-EPI $_{\text {- }}$ equation was close to the ideal diagonal at any value of BMI with a slight lower accuracy in patients with BMI $<25.0 \mathrm{~kg} / \mathrm{m}^{2}(p=0.03)$. In contrast, the MDRD was more accurate in patients with BMI $<25.0 \mathrm{~kg} / \mathrm{m}^{2}$ $(p=0.7)$ whereas it showed a trend to overprediction in subjects with BMI $\geq 25.0 \mathrm{~kg} / \mathrm{m}^{2} \quad(p=$ 0.03 and $p=0.04$ in patients with BMI $25-29 \mathrm{Kg} / \mathrm{m}^{2}$ and $\geq 30 \mathrm{Kg} / \mathrm{m}^{2}$, respectively). Finally, the $C_{\mathrm{CG}}$ formula was the less accurate up to $18.5 \mathrm{~kg} / \mathrm{m}^{2}$ ( $\mathrm{p}=$ $0.03, p=0.02$ and $\mathrm{p}=0.02$ in patients with BMI $>30$ $\mathrm{Kg} / \mathrm{m}^{2}, 25-29 \mathrm{Kg} / \mathrm{m}^{2}$ and $\geq 30 \mathrm{Kg} / \mathrm{m}^{2}$, respectively) with a tendency to over-prediction, while it was comparable to the MDRD formula when in patients with BMI $<25.0 \mathrm{~kg} / \mathrm{m}^{2}(p=0.5)$. 
Table 3 Calibration

\begin{tabular}{|c|c|c|c|c|c|c|}
\hline & & eGFR & Slope & Intercept & U statistic & Hosmer-Lemeshow \\
\hline \multirow[t]{3}{*}{$\overline{\text { All }}$} & & CKD-EPI & 0.69 & 0.12 & 0.38 & 0.40 \\
\hline & & MDRD & 1.34 & -1.06 & 0.03 & 0.04 \\
\hline & & $C G$ & 1.29 & -1.00 & 0.02 & 0.02 \\
\hline \multirow[t]{12}{*}{ eGFR } & $>=60 \mathrm{ml} / \mathrm{min} / 1.73 \mathrm{~m}^{2}$ & CKD-EPI & 0.60 & 0.15 & 0.21 & 0.58 \\
\hline & & MDRD & 1.20 & -1.16 & 0.03 & 0.02 \\
\hline & & $C G$ & 0.57 & 0.18 & 0.39 & 0.48 \\
\hline & $45-59 \mathrm{ml} / \mathrm{min} / 1.73 \mathrm{~m}^{2}$ & CKD-EPI & 0.54 & -0.14 & 0.15 & 0.78 \\
\hline & & MDRD & 1.17 & 1.56 & 0.02 & 0.03 \\
\hline & & $C G$ & 0.59 & 0.20 & 0.28 & 0.32 \\
\hline & $30-44 \mathrm{ml} / \mathrm{min} / 1.73 \mathrm{~m}^{2}$ & CKD-EPI & 0.49 & 0.18 & 0.09 & 0.39 \\
\hline & & MDRD & 0.22 & -0.16 & 0.02 & 0.04 \\
\hline & & CG & 0.28 & -0.17 & 0.03 & 0.03 \\
\hline & $<=29 \mathrm{ml} / \mathrm{min} / 1.73 \mathrm{~m}^{2}$ & CKD-EPI & 1.13 & 0.58 & 0.04 & 0.04 \\
\hline & & MDRD & 1.07 & -0.36 & 0.07 & 0.06 \\
\hline & & CG & 1.23 & -0.28 & 0.04 & 0.03 \\
\hline \multirow[t]{9}{*}{ Age } & AGE $>=60 y$ & CKD-EPI & 1.03 & 0.30 & 0.10 & 0.69 \\
\hline & & MDRD & 0.91 & -0.04 & 0.43 & 0.53 \\
\hline & & $C G$ & 0.63 & -1.35 & 0.02 & 0.03 \\
\hline & AGE 40-59 y & CKD-EPI & 0.66 & -0.24 & 0.25 & 0.41 \\
\hline & & MDRD & 0.60 & -1.77 & 0.02 & 0.03 \\
\hline & & $C G$ & 0.92 & -0.03 & 0.56 & 0.60 \\
\hline & AGE 18-39 y & CKD-EPI & 0.75 & -0.88 & 0.32 & 0.37 \\
\hline & & MDRD & 0.71 & -1.75 & 0.02 & 0.03 \\
\hline & & $C G$ & 0.59 & -1.65 & 0.01 & 0.02 \\
\hline \multirow[t]{6}{*}{ Gender } & Male & CKD-EPI & 0.85 & 0.36 & 0.20 & 0.10 \\
\hline & & MDRD & 0.70 & -1.67 & 0.03 & 0.03 \\
\hline & & CG & 0.84 & -1.43 & 0.02 & 0.03 \\
\hline & Female & CKD-EPI & 0.65 & -0.30 & 0.30 & 0.19 \\
\hline & & MDRD & 0.81 & 0.89 & 0.44 & 0.72 \\
\hline & & $C G$ & 0.79 & -1.33 & 0.03 & 0.03 \\
\hline \multirow[t]{12}{*}{ Weight } & $>=100 \mathrm{Kg}$ & CKD-EPI & 0.77 & -0.28 & 0.39 & 0.49 \\
\hline & & MDRD & 0.30 & -1.43 & 0.01 & 0.02 \\
\hline & & $C G$ & 0.50 & -1.30 & 0.02 & 0.02 \\
\hline & 80-99 Kg & CKD-EPI & 0.65 & -0.16 & 0.40 & 0.64 \\
\hline & & MDRD & 0.20 & -1.72 & 0.02 & 0.04 \\
\hline & & $C G$ & 0.46 & -1.65 & 0.01 & 0.02 \\
\hline & $60-79$ Kg & CKD-EPI & 0.56 & 0.13 & 0.19 & 0.43 \\
\hline & & MDRD & 0.70 & 0.11 & 0.31 & 0.59 \\
\hline & & $C G$ & 0.72 & -1.47 & 0.02 & 0.02 \\
\hline & $<=59 \mathrm{Kg}$ & CKD-EPI & 0.63 & 0.18 & 0.10 & 0.32 \\
\hline & & MDRD & 0.70 & 0.12 & 0.39 & 0.80 \\
\hline & & $C G$ & 0.88 & 0.20 & 0.40 & 0.47 \\
\hline \multirow[t]{2}{*}{ BMI } & $>=30 \mathrm{~kg} / \mathrm{m}^{2}$ & CKD-EPI & 0.87 & 0.13 & 0.58 & 0.67 \\
\hline & & MDRD & 0.50 & -2.07 & 0.01 & 0.04 \\
\hline
\end{tabular}


Table 3 Calibration (Continued)

\begin{tabular}{llllll}
\hline & eGFR & Slope & Intercept & U statistic & Hosmer-Lemeshow \\
\hline \multirow{2}{*}{$25-29 \mathrm{Kg} / \mathrm{m}^{2}$} & CG & 0.47 & -1.68 & 0.01 & 0.03 \\
& CKD-EPI & 0.75 & -0.39 & 0.50 & 0.57 \\
& MDRD & 0.40 & -2.05 & 0.01 & 0.03 \\
& CG & 0.40 & -1.75 & 0.02 & 0.02 \\
& CKD-EPI & 0.65 & -0.26 & 0.42 & 0.86 \\
& MDRD & 0.70 & 0.23 & 0.50 & 0.70 \\
& CG & 0.46 & -1.42 & 0.01 & 0.02 \\
& CKD-EPI & 0.85 & 0.71 & 0.16 & 0.36 \\
& MDRD & 0.80 & -0.44 & 0.48 & 0.73 \\
& CG & 0.79 & -0.37 & 0.37 & 0.50 \\
\hline
\end{tabular}

Best performance for: Slope $=1$, Intercept $=0$, non-significant $P$-values of the $U$ statistic, and Hosmer-Lemeshow test. Abbreviations: eGFR: estimated Glomerular Filtration Rate; Cl: Confidence Interval; CKD-EPI: Chronic Kidney Disease-Epidemiology Collaboration Formula; MDRD: Modification of Diet in Renal Disease Formula; $B M I:$ Body Mass Index; CG: Cockroft-Gault Formula
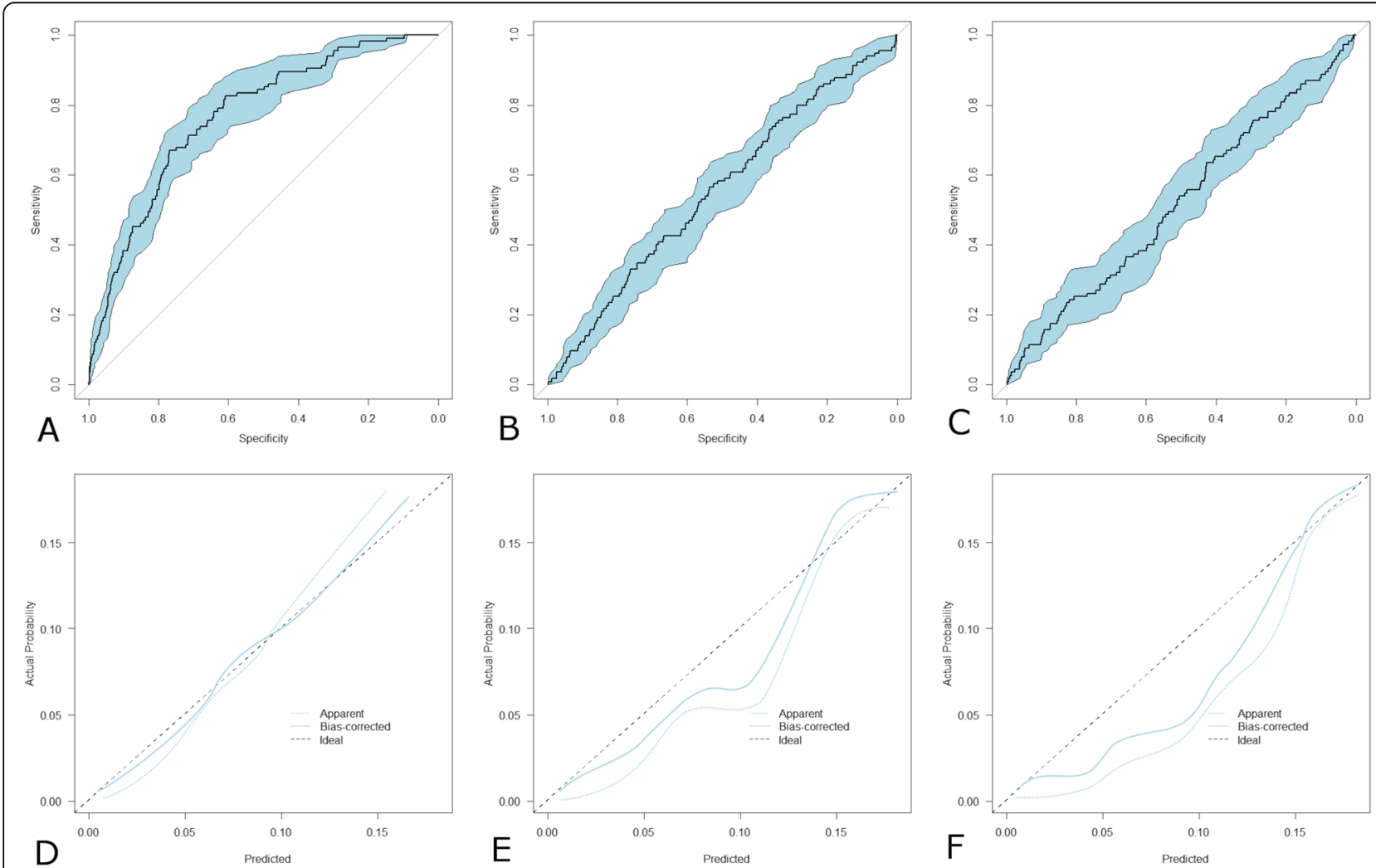

Fig. 1 Receiver operating characteristic curves with 95\% confidence intervals for eGFR $R_{C K D-E P I}$ (a) eGFR $R_{M D R D}(\mathbf{b})$ and $C_{C G}$ (c). A curve lying on the diagonal line reflects the performance of a diagnostic test that is no better than chance level. The closer is the curve to the upper left-hand corner the greater is the discriminant testing capacity. Calibration plots of eGFR $R_{C K D-E P I}(\mathbf{d})$ and eGFR represents the perfect calibration. If the line lies below the ideal curve, the EGFR formula overestimates the outcome, if it is above the ideal curve the formula underestimates the outcome 

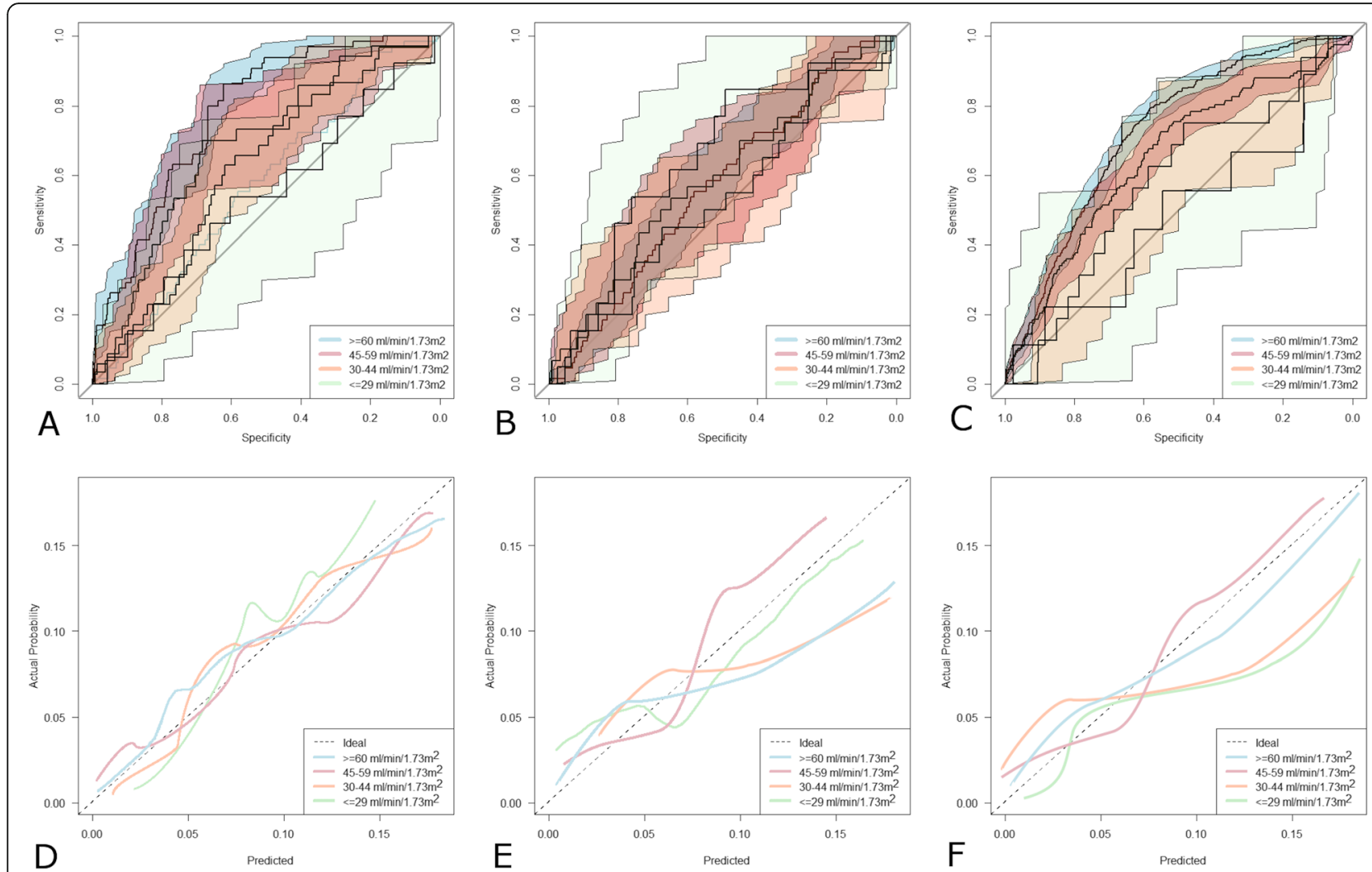

Fig. 2 Patients Stratified by Renal Function. Receiver operating characteristic curves with 95\% confidence intervals for eGFR (b). and $\mathrm{C}_{\mathrm{CG}}(\mathbf{c})$. Colored curve above the diagonal line perform progressively better the closer they are to the upper left-hand corner. Calibration plots of eGFR CKD-EPI $_{(\mathbf{d}) \text { eGFR }}$ MDRD $(\mathbf{e})$ and $\mathrm{C}_{\mathrm{CG}}(\mathbf{f})$. Lines below the ideal curve (dotted line) overestimate the outcome, if they lie above the ideal curve the outcome is underestimated

\section{Discussion}

Patients with CAD and renal disease have a dismal prognosis [34, 35]. In addition, estimated glomerular filtration rate (eGFR) has a major impact on the outcome of patients undergoing coronary revascularization, either percutaneous coronary intervention or coronary artery bypass grafting (CABG) $[10,36]$.

Reduced erythropoietin synthesis and consequent anemia and reduced $1,25(\mathrm{OH})$ vitamin D production, associated with increased parathyroid hormone levels and higher prevalence of vascular calcification and arteriosclerosis have been reported to explain the association between renal dysfunction and cardiovascular events [37, 38].

In addition, patients with reduced or impaired renal function face additional challenges in the setting of CABG for several reasons: 1) Concomitant factors such as including advanced age, low ejection fraction, history myocardial infarction, and stroke which are themselves determinants of poor outcomes [39]. 2) Detrimental cardiovascular effects by oxidative stress and high levels of homocysteine, hyperuricemia, hypercalcemia, and uremia associated with reduced renal function [40, 41].
3) Higher incidence of multivessel disease and microvessel disease in such patients $[2,11]$.

However, little is known whether eGFRs calculated with different formulas have comparable predictive value on post-CABG mortality.

In our recent paper [42] we had shown that the eGFR $_{\text {CKD-EPI }}$ equation led to categorization with a significantly lower number of patients at risk for postCABG complications and with cut-off values of eGFR $\mathrm{CK}_{\text {- }}$ D-EPI predicting early and late events significantly lower than accepted prediction threshold values for postCABG unfavorable events [2, 41, 43].

In the present study our study we assessed the performance, in terms of discrimination and calibration, of the MDRD, CG formulas and CKD-EPI equations in predicting mortality after CABG in the whole patient population and across different subgroups of patients defined by eGFR, age, gender and body size.

The main findings of our study can be summarized as follows:

1) The overall performance of eGFR $\mathrm{CKD}_{\text {-EPI }}$ in prediction of post-CABG death is significantly 

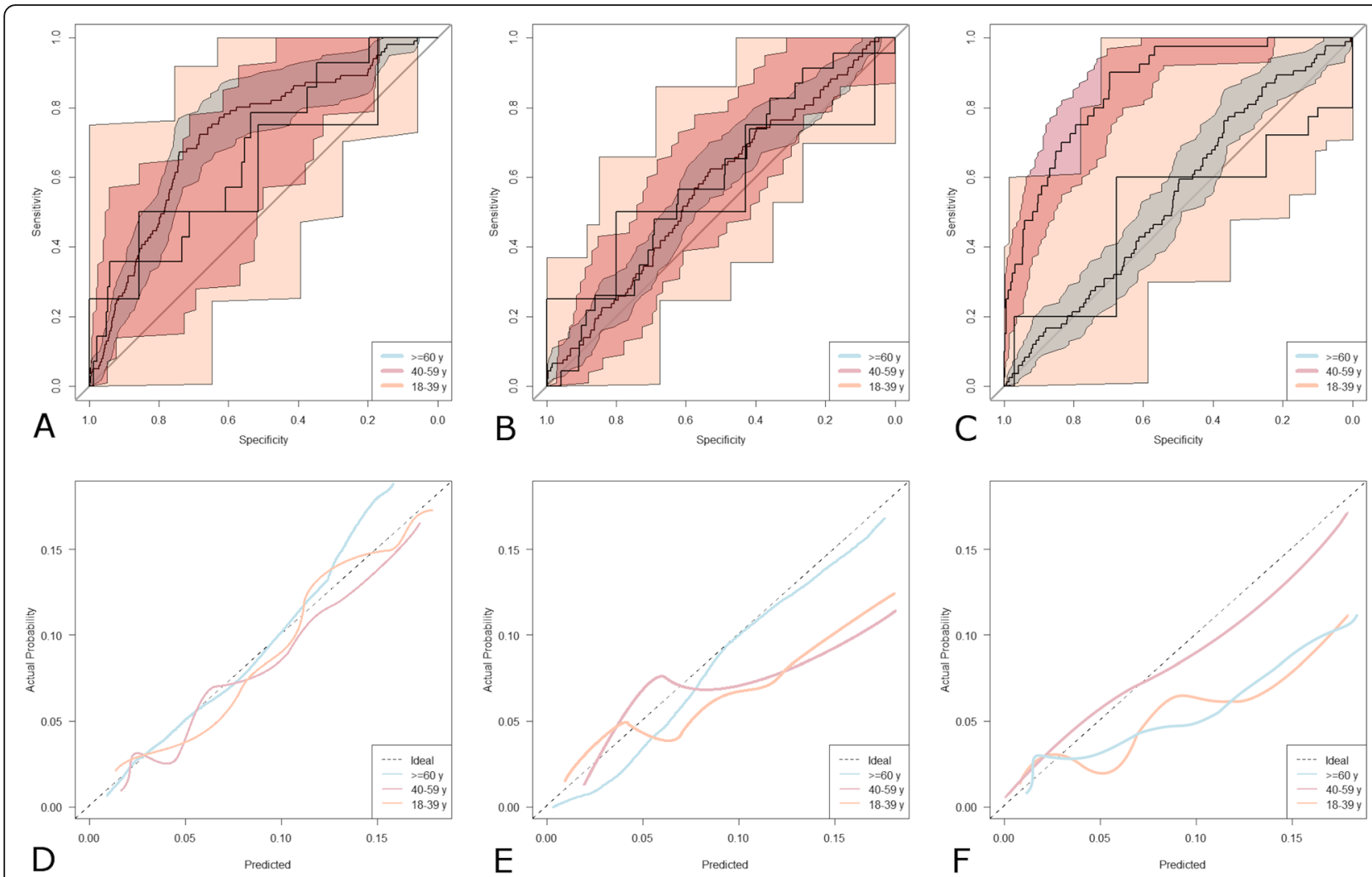

Fig. 3 Patients Stratified by Age. Receiver operating characteristic curves with 95\% confidence intervals for eGFR CKD-EPI $_{\text {(a) }}$ eGFFRDRD (b) and $C_{C G}$ (c) stratified by age. Colored curve above the diagonal line perform progressively better the closer they are to the upper left-hand corner.

Calibration plots of eGFR $\mathrm{CKD}_{\text {-EPI }}(\mathbf{d})$ and eGFR $\mathrm{MDRD}_{\mathrm{M}}(\mathbf{e})$ and $\mathrm{C}_{\mathrm{CG}}(\mathbf{f})$. Lines below the ideal curve (dotted line) overestimate the outcome, if they lie above the ideal curve the outcome is underestimated

superior to both eGFR $\mathrm{MDRD}$ and CG formulas and its calibration curve is close to the ideal prediction over a wide range of thresholds for mortality risk prediction whereas the MDRD and CG equations, show a general trend towards over-prediction.

2) The CKD-EPI equation gave the best overall accuracy and agreement after classification in subgroups of GFR. Furthermore, it had a greater accuracy in patients with an eGFR $>30 \mathrm{ml} / \mathrm{min} / 1.73 \mathrm{~m}^{2}$ whereas it showed a trend towards under-predicting mortality when the eGFR fell below $30 \mathrm{ml} / \mathrm{min} / 1.73 \mathrm{~m}^{2}$. In

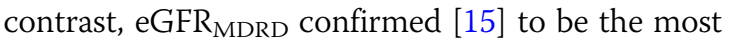
reliable in patients with highly compromised renal function whilst $\mathrm{C}_{\mathrm{CG}}$ showed comparable performance of eGFR CKD-EPI $_{\text {when }}$ eGFR was $>44 \mathrm{ml} / \mathrm{min} /$ $1.73 \mathrm{~m}^{2}$.

3) Previous studies have demonstrated that the performance of eGFR equations depends on the stage of CKD [44], thus being greatly influenced by the value of glomerular filtration rate [15]. In addition, the MDRD equation resulted in imprecise and underestimates of eGFR at higher renal function levels [45]. In our experience, the accurateness of the CKD- EPI formula in predicting post-CABG mortality was independent of age and gender whereas eGFR $\mathrm{MDRD}_{\mathrm{S}}$ overestimated the prediction in younger patients and in men while it was accurate in women and patients $\geq 60$ years and $C_{C G}$ tended to over-prediction in the $\geq 60$ year- and 18 39 year-subgroups and in both genders. This might be related to the uncertain reliability of these formulas in reflecting the true renal function $[46,47]$

4) Since all three formulas rely on serum creatinine as the indicator for the rate of glomerular filtration and because serum creatinine correlates with muscle mass and nutritional status, the performance of the formulas might be influenced by body composition. This was assessed by studying the influence of body mass or BMI on eGFR, which, in our experience, did not affect the CKD-EPI equation whose calibration curve was close to the ideal diagonal at any value of BMI. In contrast, the MDRD was accurate only in overweight patients and those with body mass $\geq 30.0 \mathrm{~kg} / \mathrm{m} 2$. These results are in accordance with Michels et al. [15] who found that MDRD provided greatest accuracy in 

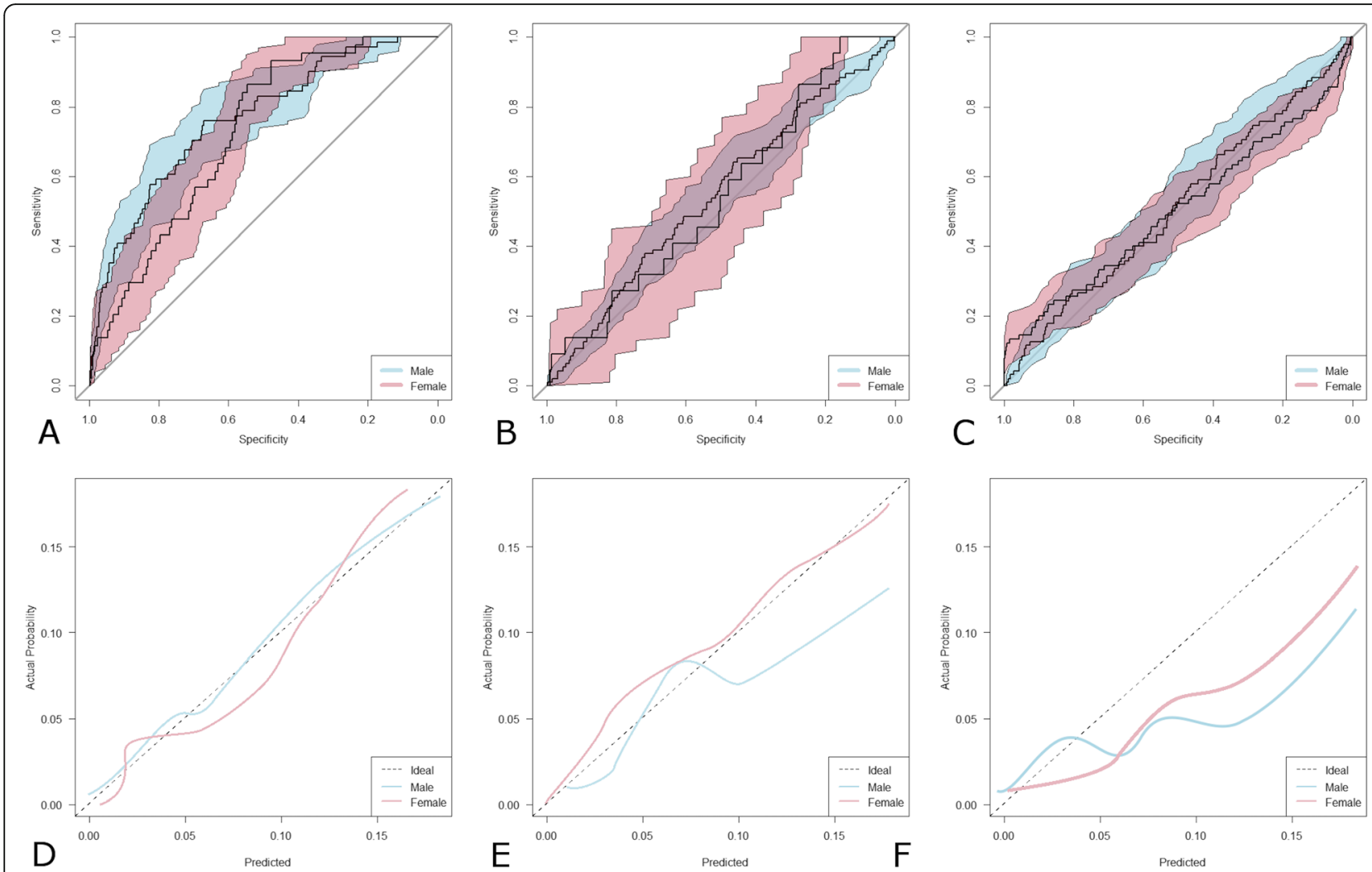

Fig. 4 Patients Stratified by Gender. Receiver operating characteristic curves with 95\% confidence intervals for eGFR $R_{C K D-E P I}(\mathbf{a})$ eGFR $R_{M D R D}$ (b) and $\mathrm{C}_{\mathrm{CG}}(\mathbf{c})$. . Colored curve above the diagonal line perform progressively better the closer they are to the upper left-hand corner. Calibration plots of eGFR $_{C K D \text {-EPI }}(\mathbf{d})$ eGFR $R_{M D R D}(\mathbf{e})$ and $C_{C G}(\mathbf{f})$. Lines below the ideal curve (dotted line) overestimate the outcome, if they lie above the ideal curve the outcome is underestimated

defining renal function $(97.0 \%)$ in subjects with the highest body weight whereas other studies showed no relation or positive correlation concluding that no creatinine-based method is reliable in the obese [48]. Lastly, the CCG formula was the least accurate up to $18.5 \mathrm{~kg} / \mathrm{m} 2$ while it was comparable to the MDRD formula in smaller patients.

Renal function is regularly included in all risk stratification models in cardiac surgical patients. Two wellrecognized risk models assess cardiovascular outcomes of patients undergoing CABG: the EuroSCORE and the Society of Thoracic Surgeons (STS) National Adult Cardiac Database [49]. The first employs eGFR calculated with $C_{\text {CG }}$ formula and value ranges that are not concordant with $\mathrm{Na}$ tional Kidney Foundation recommendations [50] whereas the STS risk score incorporates a continuous parameter for serum creatinine and a binary variable for hemodialysis [51]. Based on KDIGO clinical practice Guidelines [8] and previous evidence [15], it would be of great interest to test, in a broad patient population, the eGFR $\mathrm{CKD}_{\text {-EPI }}$ formula incorporated into CABG risk prediction algorithm, reestimating the weight for all the variables in the predictive tool, to compare the predictive performance of such a model to algorithms currently in use. At this point, in the absence of validation studies, it is impossible to understand

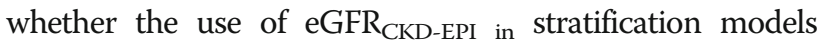
would make a valuable contribution to improve the predictive value of the algorithm. Further research is warranted.

\section{Study limitations}

This study has some limitation that should be highlighted. Firstly, its retrospective nature makes it impossible to draw final conclusions. Secondly, the population is relatively small, and assessment of the equations was carried out in a restricted study population (i.e. post CABG patients), limiting extrapolation of findings to other cohorts such as myocardial infarction, heart failure etc. Thirdly, the patient population has several variations from most CABG profiles: low number of female, low incidence of adult onset diabetes mellitus, unstable angina and MI < 30 days and high number of patients receiving 1-2 grafts. Fourthly, patients with associated procedures were excluded and this could introduce another bias. We wanted to test the three indices 

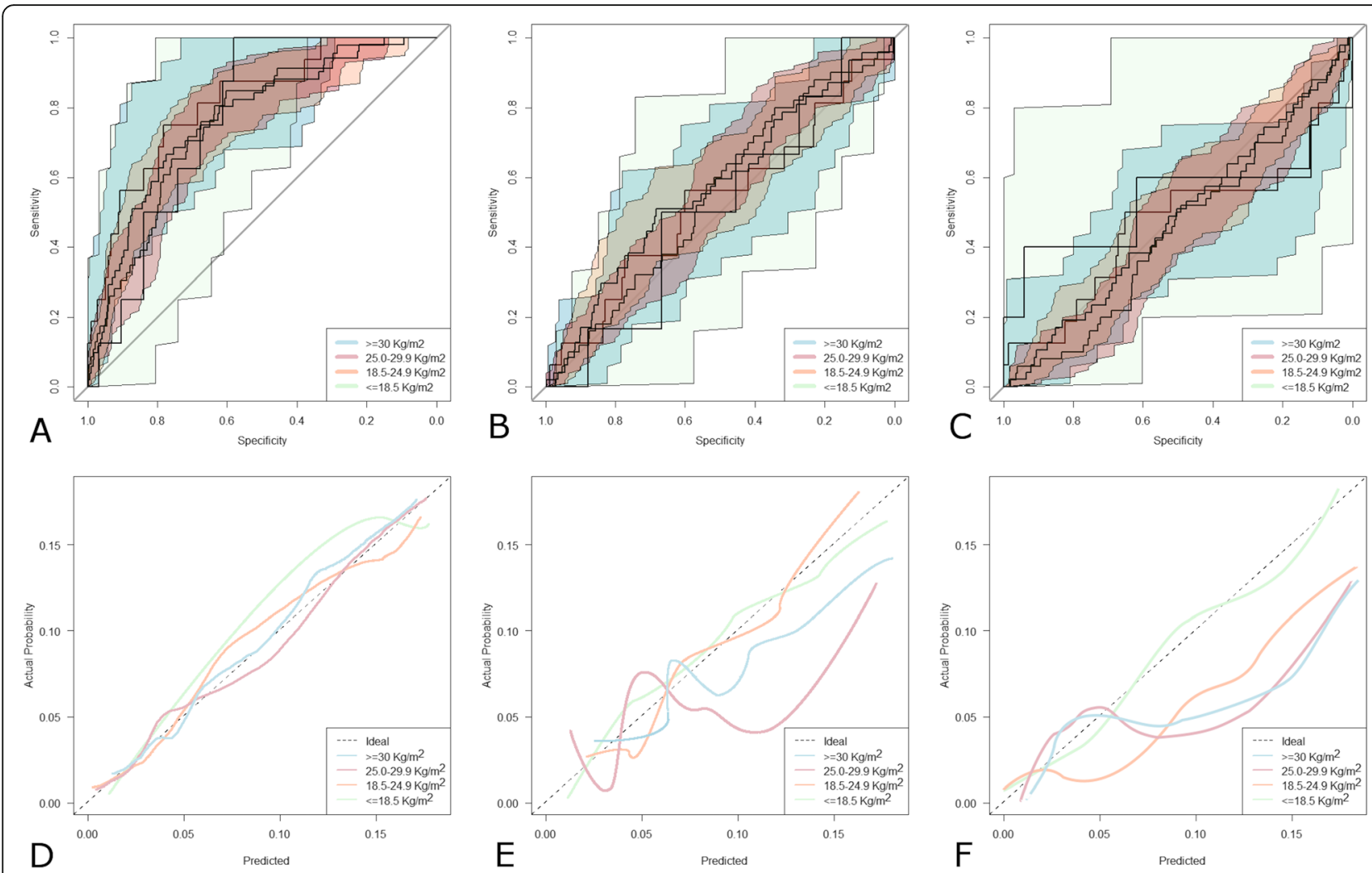

Fig. 5 Patients Stratified by Body Mass Index (BMI). Receiver operating characteristic curves with 95\% confidence intervals for eGFR eGFR $R_{M D R D}(\mathbf{b})$. and $C_{C G}(\mathbf{c})$. . Colored curve above the diagonal line perform progressively better the closer they are to the upper left-hand corner. Calibration plots of eGFR $\mathrm{CKD}_{\text {EPI }}(\mathbf{d})$ eGFR $_{\mathrm{MDRD}}(\mathbf{e})$ and $\mathrm{C}_{\mathrm{CG}}(\mathbf{f})$. Lines below the ideal curve (dotted line) overestimate the outcome, if they lie above the ideal curve the outcome is underestimated

excluding as much as possible confounding factors. Fifthly, preoperative eGFR was calculated on a single measurement and therefore susceptible of being influenced by cardiac function and therapy. Sixthly, preoperative renal function was unknown which could have postCABG survival. Seventhly, eGFR ${ }_{\text {CKD-EPI }}$ still has the limitation of being related to muscle mass, thus other filtration markers such as serum cystatin might have helped us in overcoming this issue. Eighthly, data presented in this paper did not say anything about which equation is the better predictor of true GFR, but it was beyond the aim of the paper that was explore which eGFR formula is the best predictor of mortality. The two things may go hand-inhand, but this cannot be concluded from the existing data and it will be object of upcoming research. Finally, neither we compare the performance of the three formulae within specific risk scores, nor did not test the performance of eGFR $_{\text {CKD-EPI }}$ on postoperative renal failure but these were beyond the aim of the present study.

\section{Conclusions}

In general, CKD-EPI gives the best prediction of death after CABG with unsatisfactory accuracy and calibration only in patients with severe CKD. In contrast, the CG and MDRD equations were inaccurate in predicting mortality in a clinically significant proportion of patients. eGFR $_{\text {CKD-EPI }}$ should be incorporated into CABG riskassessment algorithms to provide patients and their family members the most accurate risk prediction.

\section{Supplementary information}

Supplementary information accompanies this paper at https://doi.org/10. 1186/s12882-019-1564-y.

Additional file 1. Missing Data. Frequencies of missing data.

\section{Abbreviations}

AUC: Area under curve; BMI: Body mass index; BSA: Body surface area; CABG: Coronary artery bypass grafting; CCG: Creatinine Clearance estimated by Cockroft-Gault formula; CKD: Chronic kidney disease AUC: Area under curve; eGFR

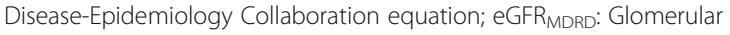
Filtration Rate estimated by the Modification in Diet in Renal Disease; FCS: Fully conditional specification; H-L: Hosmer-Lemeshow goodness-of-fit test; KDIGO: Kidney Disease Improving Global Outcomes; KDOQI: Kidney Disease Outcomes Quality Initiative; MI: Multiple imputation; OPCAB: OffPump Coronary Artery Bypass; ROC: Receiver operating characteristic 


\section{Acknowledgments}

We gratefully acknowledge Judith Wilson for the English Editing of the Manuscript.

\section{Authors' contributions}

SG, FB, conception of the study, writing; SDP, Collection of data; SG FL OP, analysis of data, DJ Revision of the manuscript for important intellectual content; MG, MB Conception of the work, Data interpretation, Final revision All authors have read and approved the manuscript.

\section{Funding}

The authors received no financial support for the research and/or authorship of this article.

\section{Availability of data and materials}

The datasets used and/or analysed during the current study are available from Orlando Parise on reasonable request.

\section{Ethics approval and consent to participate}

Ethical Committee approval was waived due to the retrospective analysis of the study according to National Laws regulating observational retrospective studies (Italian law nr.11960, released on 13/07/2004). However, patients gave their written informed consent or the treatment of their data for scientific purposes

\section{Consent for publication}

Not Applicable.

\section{Competing interests}

The authors declare no conflicts of interest.

\section{Author details}

${ }^{1}$ Cardiothoracic Department, Maastricht University Hospital, Florence, Italy. ${ }^{2}$ Department of Cardiothoracic Surgery, Cardiovascular Research Institute Maastricht University, Universiteitssingel 50, 6229, ER, Maastricht, The Netherlands. ${ }^{3}$ Cardiothoracovascular Department, Careggi University Hospital, Florence, Italy. ${ }^{4}$ ANMCO Research Center of Heart Care, Florence, Italy. ${ }^{5}$ Department of Cardiovascular Surgery, S. Croce Hospital, Cuneo, Italy. ${ }^{6}$ Cardiology Garibaldi-Nesima Hospital, Catania, Italy.

\section{Received: 5 December 2018 Accepted: 26 September 2019} Published online: 16 October 2019

\section{References}

1. Cooper WA, O'Brien SM, Thourani VH, Guyton RA, Bridges CR, Szczech LA, Petersen R, Peterson ED. Impact of renal dysfunction on outcomes of coronary artery bypass surgery: results from the Society of Thoracic Surgeons National Adult Cardiac Database. Circulation. 2006;113(8):1063-70.

2. Hillis GS, Croal BL, Buchan KG, El-Shafei H, Gibson G, Jeffrey RR, Millar CG, Prescott GJ, Cuthbertson BH. Renal function and outcome from coronary artery bypass grafting: impact on mortality after a 2.3-year follow-up. Circulation. 2006;113(8):1056-62.

3. van Straten AHM, Soliman Hamad MA, van Zundert AAJ, Martens EJ, Schönberger JPAM, de Wolf AM. Preoperative renal function as a predictor of survival after coronary artery bypass grafting: comparison with a matched general population. J Thorac Cardiovasc Surg. 2009;138(4):971-6.

4. Hori D, Yamaguchi A, Adachi H. Coronary artery bypass surgery in end-stage renal disease patients. Ann Vasc Dis. 2017:10(2):79-87.

5. Sarafidis PA, Bakris GL. Level of kidney function determines cardiovascular fate after coronary bypass graft surgery. Circulation. 2006;113(8):1046-7.

6. Goolsby MJ. National Kidney Foundation guidelines for chronic kidney disease: evaluation, classification, and stratification. J Am Acad Nurse Pract. 2002;14(6):238-42.

7. Lamb EJ, Levey AS, Stevens PE. The kidney disease improving global outcomes (KDIGO) guideline update for chronic kidney disease: evolution not revolution. Clin Chem. 2013;59(3):462-5.

8. Inker LA, Astor BC, Fox CH, Isakova T, Lash JP, Peralta CA, Kurella Tamura M, Feldman HI. KDOQI US commentary on the $2012 \mathrm{KDIGO}$ clinical practice guideline for the evaluation and management of CKD. Am J Kidney Dis. 2014;63(5):713-35.
9. Holzmann MJ, Sartipy U. Relation between preoperative renal dysfunction and cardiovascular events (stroke, myocardial infarction, or heart failure or death) within three months of isolated coronary artery bypass grafting. Am J Cardiol. 2013;112(9):1342-6.

10. Kangasniemi OP, Mahar MA, Rasinaho E, Satomaa A, Tiozzo V, Lepojanvi M, Biancari F. Impact of estimated glomerular filtration rate on the 15-year outcome after coronary artery bypass surgery. Eur J Cardiothorac Surg. 2008;33(2):198-202.

11. Domoto S, Tagusari O, Nakamura Y, Takai H, Seike Y, Ito Y, Shibuya Y, Shikata F. Preoperative estimated glomerular filtration rate as a significant predictor of long-term outcomes after coronary artery bypass grafting in Japanese patients. Gen Thorac Cardiovasc Surg. 2014;62(2):95-102.

12. Charytan DM, Yang SS, McGurk S, Rawn J. Long and short-term outcomes following coronary artery bypass grafting in patients with and without chronic kidney disease. Nephrol Dial Transplant. 2010;25(11):3654-63.

13. Liu JY, Birkmeyer NJ, Sanders JH, Morton JR, Henriques HF, Lahey SJ, Dow RW, Maloney C, DiScipio AW, Clough R, et al. Risks of morbidity and mortality in dialysis patients undergoing coronary artery bypass surgery. Northern New England cardiovascular disease study group. Circulation. 2000;102(24):2973-7.

14. Zhang Q, Ma CS, Nie SP, Du X, Lv Q, Kang JP, Zhang Y, Hu R, Jia CQ, Liu XM, et al. Prevalence and impact of renal insufficiency on clinical outcomes of patients undergoing coronary revascularization. Circ J. 2007;71(8):1299-304.

15. Michels WM, Grootendorst DC, Verduijn M, Elliott EG, Dekker FW, Krediet RT. Performance of the Cockcroft-Gault, MDRD, and new CKD-EPI formulas in relation to GFR, age, and body size. Clin J Am Soc Nephrol. 2010;5(6):1003-9.

16. von Elm E, Altman DG, Egger M, Pocock SJ, Gøtzsche PC, Vandenbroucke $J P$. The strengthening the reporting of observational studies in epidemiology (STROBE) statement: guidelines for reporting observational studies. J Clin Epidemiol. 2008;61(4):344-9.

17. Gelsomino S, Del Pace S, Parise O, Caciolli S, Matteucci F, Fradella G, Bonacchi M, Fusco S, Luca F, Marchionni N. Impact of renal function impairment assessed by CKDEPI estimated glomerular filtration rate on early and late outcomes after coronary artery bypass grafting. Int J Cardiol. 2017;227:778-87.

18. Vassalotti JA, Centor R, Turner BJ, Greer RC, Choi M, Sequist TD. National Kidney Foundation kidney disease outcomes quality l: practical approach to detection and Management of Chronic Kidney Disease for the primary care clinician. Am J Med. 2016;129(2):153-62 e157.

19. Cockcroft DW, Gault MH. Prediction of creatinine clearance from serum creatinine. Nephron. 1976;16(1):31-41.

20. Levey AS, Coresh J, Greene T, Stevens LA, Zhang YL, Hendriksen S, Kusek JW, Van Lente F. Chronic kidney disease epidemiology C: using standardized serum creatinine values in the modification of diet in renal disease study equation for estimating glomerular filtration rate. Ann Intern Med. 2006;145(4):247-54

21. Levey AS, Stevens LA, Schmid CH, Zhang YL, Castro AF 3rd, Feldman HI, Kusek JW, Eggers P, Van Lente F, Greene T, et al. A new equation to estimate glomerular filtration rate. Ann Intern Med. 2009;150(9):604-12.

22. Du Bois D, Du Bois EF. A formula to estimate the approximate surface area if height and weight be known. Nutrition 1989. 1916;5(5):303-11 discussion 312-303.

23. Bartfay E, Bartfay WJ. Accuracy assessment of prediction in patient outcomes. J Eval Clin Pract. 2008:14(1):1-10.

24. Harrel FE, Lee KL, Mark DB. Multivariable prognostic models: issues in developing models, evaluating assumptions and adequacy and measuring and reducing errors. Stat Med. 1996;15:361-87.

25. Cook NR. Use and misuse of the receiver operating characteristic curve in risk prediction. Circulation. 2007;115(7):928-35.

26. Sing T, Sander O, Beerenwinkel N, Lengauer T. ROCR: visualizing classifier performance in R. Bioinformatics. 2005;21(20):3940-1.

27. Robin X, Turck N, Hainard A, Tiberti N, Lisacek F, Sanchez J-C, Müller M. PROC: an open-source package for R and S+ to analyze and compare ROC curves. BMC Bioinformatics. 2011;12(1):77.

28. Harrel FE. Regression Modeling Strategies. New York: Springer; 2001.

29. Barili F, Pacini D, Capo A, Rasovic O, Grossi C, Alamanni F, Di Bartolomeo R, Parolari A. Does EuroSCORE II perform better than its original versions? A multicentre validation study. Eur Heart J. 2012;34(1):22-9.

30. Steyerberg EW, Vickers AJ, Cook NR, Gerds T, Gonen M, Obuchowski N, Pencina MJ, Kattan MW. Assessing the performance of prediction models: a framework for traditional and novel measures. Epidemiology. 2010;21(1):128-38.

31. Afilalo J, Steele R, Manning WJ, Khabbaz KR, Rudski LG, Langlois Y, Morin JF, Picard MH. Derivation and validation of prognosis-based age cutoffs to define elderly in cardiac surgeny. Circ Cardiovasc Qual Outcomes. 2016;9(4):424-31. 
32. Executive summary of the clinical guidelines on the identification, evaluation, and treatment of overweight and obesity in adults. Arch Intern Med 1998, 158(17):1855-1867.

33. Liu Y, De A. Multiple imputation by fully conditional specification for dealing with missing data in a large epidemiologic study. Int I Stat Med Research. 2015;4(3):287-95.

34. Go AS, Chertow GM, Fan D, McCulloch CE, Hsu C-y: Chronic kidney disease and the risks of death, cardiovascular events, and hospitalization. N Engl J Med 2004, 351(13):1296-1305.

35. Manjunath G, Tighiouart H, Ibrahim H, MacLeod B, Salem DN, Griffith JL, Coresh J, Levey AS, Sarnak MJ. Level of kidney function as a risk factor for atherosclerotic cardiovascular outcomes in the community. J Am Coll Cardiol. 2003;41(1):47-55.

36. Berger AK, Herzog CA. CABG in CKD: untangling the letters of risk. Nephrol Dial Transplant. 2010;25(11):3477-9.

37. Cozzolino M, Brancaccio D, Gallieni M, Slatopolsky E. Pathogenesis of vascular calcification in chronic kidney disease. Kidney Int. 2005;68(2):429-36.

38. Strippoli GFM, Craig JC, Manno C, Schena FP. Hemoglobin targets for the Anemia of chronic kidney disease: a meta-analysis of randomized, controlled trials. J Am Soc Nephrol. 2004;15(12):3154-65.

39. Sarnak MJ, Levey AS, Schoolwerth AC, Coresh J, Culleton B, Hamm LL, McCullough PA, Kasiske BL, Kelepouris E, Klag MJ, et al. Kidney disease as a risk factor for development of cardiovascular disease: a statement from the American Heart Association councils on kidney in cardiovascular disease, high blood pressure research, clinical cardiology, and epidemiology and prevention. Hypertension. 2003;42(5):1050-65.

40. Jardine AG, McLaughlin K. Cardiovascular complications of renal disease. Heart. 2001;86(4):459-66.

41. Anavekar NS, McMurray JJ, Velazquez EJ, Solomon SD, Kober L, Rouleau JL, White HD, Nordlander R, Maggioni A, Dickstein K, et al. Relation between renal dysfunction and cardiovascular outcomes after myocardial infarction. N Engl J Med. 2004;351(13):1285-95.

42. Gelsomino S, Del Pace S, Parise O, Caciolli S, Matteucci F, Fradella G, Bonacchi M, Fusco S, Luca F, Marchionni N. Impact of renal function impairment assessed by CKDEPI estimated glomerular filtration rate on early and late outcomes after coronary artery bypass grafting. Int J Cardiol. 2017;227:778-87.

43. Go AS, Chertow GM, Fan D, McCulloch CE, Hsu CY. Chronic kidney disease and the risks of death, cardiovascular events, and hospitalization. N Engl J Med. 2004;351(13):1296-305.

44. Froissart M, Rossert J, Jacquot C, Paillard M, Houillier P. Predictive performance of the modification of diet in renal disease and Cockcroft-Gault equations for estimating renal function. J Am Soc Nephrol. 2005;16(3):763-73.

45. Poggio ED, Nef PC, Wang X, Greene T, Van Lente F, Dennis WW, Hall PM. Performance of the Cockcroft-Gault and modification of diet in renal disease equations in estimating GFR in ill hospitalized patients. Am J Kidney Dis. 2005:46(2):242-52.

46. Roberts GW, Ibsen PM, Schioler CT. Modified diet in renal disease method overestimates renal function in selected elderly patients. Age Ageing. 2009; 38(6):698-703.

47. Murata K, Baumann NA, Saenger AK, Larson TS, Rule AD, Lieske JC. Relative performance of the MDRD and CKD-EPI equations for estimating glomerular filtration rate among patients with varied clinical presentations. Clin J Am Soc Nephrol. 2011;6(8):1963-72.

48. Bird NJ, Peters C, Michell AR, Peters AM. Reliability of the MDRD method for estimating glomerular filtration rate in relation to gender, body mass index and extracellular fluid volume. Eur J Clin Investig. 2008;38(7):486-93.

49. Nilsson J, Algotsson L, Höglund P, Lührs C, Brandt J. Early mortality in coronary bypass surgery: the EuroSCORE versus the Society of Thoracic Surgeons risk algorithm. Ann Thorac Surg. 2004;77(4):1235-9.

50. Zalewska-Adamiec M, Bachorzewska-Gajewska H, Malyszko J, Malyszko JS, Kralisz P, Tomaszuk-Kazberuk A, Hirnle T, Dobrzycki S. Chronic kidney disease in patients with significant left main coronary artery disease qualified for coronary artery bypass graft operation. Arch Med Sci. 2015;11(2):446-52.

51. Welke KF, Ferguson TB Jr, Coombs LP, Dokholyan RS, Murray CJ, Schrader MA, Peterson ED. Validity of the Society of Thoracic Surgeons National Adult Cardiac Surgery Database. Ann Thorac Surg. 2004;77(4):1137-9.

\section{Publisher's Note}

Springer Nature remains neutral with regard to jurisdictional claims in published maps and institutional affiliations.

\section{Ready to submit your research? Choose BMC and benefit from:}

- fast, convenient online submission

- thorough peer review by experienced researchers in your field

- rapid publication on acceptance

- support for research data, including large and complex data types

- gold Open Access which fosters wider collaboration and increased citations

- maximum visibility for your research: over $100 \mathrm{M}$ website views per year

At $\mathrm{BMC}$, research is always in progress.

Learn more biomedcentral.com/submissions 